\title{
Flight Path Planning for UAV Atmospheric Energy Harvesting Using Heuristic Search
}

\author{
Anjan Chakrabarty* \\ Jack W. Langelaan ${ }^{\dagger}$ \\ The Pennsylvania State University, University Park, PA 16802, USA
}

\begin{abstract}
This paper presents an approach to planning long distance autonomous soaring trajectories for small uninhabited aerial vehicles harvesting energy from the atmosphere. An $A^{*}$ algorithm is used with a cost function which is the weighted sum of energy required and distance to goal. The effect of varying the weight parameter on the flight paths is explored. The required initial energy for varying weight is examined, and the results are compared with a wavefront expansion planning algorithm. The weight is selected based on maximum energy utilization that is available from the atmosphere and minimizing time to reach the goal. Optimal weight is selected based on simulation results and the performance of $A^{*}$ is studied for a realistic wind field. Optimal energy efficient routes are predicted from a given wind field data.
\end{abstract}

\section{Introduction}

Small Uninhabited Aerial Vehicles (UAVs, here "small" means hand-launchable) are limited in range and endurance because of their limitation of fuel that can be carried on board. Moreover, the best L/D attainable for small UAVs is typically much smaller than for larger aircraft. While improvements in battery technology will enable longer duration missions, immediate performance improvements can achieved by harvesting energy available from the atmosphere. Further, extremely long duration and long distance flights can be performed if energy is harvested from the atmosphere. Indeed, large birds such as hawks and eagles as well as human sailplane and hang glider pilots routinely exploit the energy available from updrafts of air to fly for hundreds of kilometers without flapping wings or the use of engines.

Vertical air motion or updrafts is the main source from which energy can be harvested. There are three reasons for the cause of updrafts: uneven heating of the ground, which produces buoyant instabilities known as thermals; long period oscillations of the atmosphere, generally called wave, which occurs in the lee of large mountain ranges; and orographic lift, where wind is deflected by the slopes of hills and mountains. Ridge lift and wave can be predicted based on the local wind field and terrain, thus this phenomenon can be used for long distance trajectory planning. Energy harvesting from these relatively long-duration phenomena is called static soaring.

Autonomous static soaring is fast becoming an active area of research. Several authors have addressed the optimal static soaring trajectory problem in the context of soaring competition. The MacCready problem, ${ }^{1,2}$ the final glide problem, ${ }^{3}$ and "Dolphin" flight along regions of alternating lift and $\operatorname{sink}^{4-6}$ all address optimal static soaring including optimal speed to fly between thermals of known strength. de Jong ${ }^{7}$ describes a geometric approach to trajectory optimization. Most of this research is limited by known lift distribution (e.g. sinusoidally varying lift ${ }^{8}$ or "square wave" lift $^{9}$ ) and generally do not consider the effects of horizontal wind components.

Recent works including simulation results of thermal flight are reported by Allen $(2005)^{10}$ and flight test results are presented in Allen (2007). ${ }^{11}$ Autonomous thermal soaring has also been addressed by Edwards. ${ }^{12}$ However, because thermals are unpredictable trajectory planning is not addressed.

\footnotetext{
${ }^{*}$ Graduate Student, Department of Aerospace Engineering, Student Member AIAA

${ }^{\dagger}$ Assistant Professor, Department of Aerospace Engineering, Senior Member AIAA.
} 
A genetic algorithm approach to flight path planning wind has been addressed by Rubio. ${ }^{13}$ Jardin uses neighboring optimal control ${ }^{14}$ for wind routing problems. But none of these approaches uses the fact that energy can be harvested in regions of upward moving air.

The focus of this paper is on planning long-distance soaring trajectories which harvest energy available from a known wind field (this may be obtained from predictions generated using meteorological forecasting tools such as $\mathrm{MM} 5^{15}$ ). Previous research addressed this problem using a probabilistic road map approach, ${ }^{16}$ using gradient-based optimization, ${ }^{17}$ and by generating an energy map (i.e. a map of the minimum initial energy required to reach the goal) from all points in the environment. ${ }^{18}$

The energy map approach uses wavefront expansion from the goal to compute minimum energy paths. Flight paths are restricted to always proceed towards the goal, which enables fast solutions at the cost of not allowing paths with potentially less required initial energy but which require some flight away from the goal. The energy map thus provides an upper bound on minimum energy required to reach the goal from anywhere in the environment. In this paper the flight-to-goal constraint is relaxed and an $\mathrm{A}^{*}$ algorithm is used to find minimum cost paths to goal, where the cost is a combination of required energy and remaining distance to goal. Finding a "good" cost function to optimize time to reach the goal and energy utilization is the key of using heuristic search in soaring application. This paper discusses the use of $\mathrm{A}^{*}$ in this application (namely energy harvesting flight). This paper uses wind fields typical of mountainous regions (such as the Appalachians of Central Pennsylvania, the Rocky Mountains or the Andes) for discussion purposes. However, the methods developed here are generally applicable to any case where a prediction of wind field is available.

The remainder of the paper is organized as follows. Section II discusses path planning based on $A^{*}$ for energy harvesting flight. Section III describes the method in which the energy required for each transition and hence the cost function for the path planner is calculated. Section IV describes the planning approach for two sample wind fields: a simplified convection environment and a simplified ridge environment. Section $\mathrm{V}$ demonstrates the performance of $\mathrm{A}^{*}$ application for a wind field computed using a high-fidelity meteorology prediction tool, and finally Section VI presents the concluding remarks.

\section{Path Planning based on Heuristic Search}

Graph based techniques have been successfully implemented in many robotic application for path planning. The robot's configuration space is divided into finite number of regions (or nodes), and the planning problem is reduced to finding a sequence of of neighboring nodes between the start and goal nodes. In most cases a fixed cost function for transition between nodes in the graph is considered and the vehicle speed is kept constant. However in aircraft planning scenarios both environmental and control parameters effect the energy required for a particular transition. For example a head wind as well as flying at non-optimal airspeed will increase the energy cost of a transition.

Figure 1 shows a schematic of graph based planning applied to autonomous soaring. Contours show the vertical component of wind, with red showing upward motion (allowing energy harvesting) and

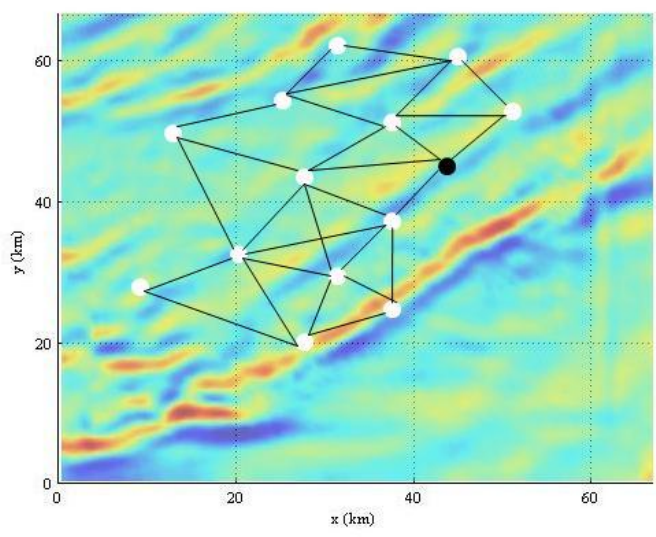

Figure 1. Schematic of graph-based planning for autonomous soaring over a wind field. blue showing downward motion. The environment is first seeded with waypoints (or nodes) and edges. This set of nodes $i=\{i=0 \ldots m\}$ (with $i=0$ denoting the goal) and edges $i j=\{i=0 \ldots m, j=0 \ldots m\}$ connecting nodes define the allowable paths to the goal. Each edge $i j$ is assigned a constant wind vector $\mathbf{w}_{i j}$ with components $w_{x, i j}, w_{y, i j}$, and $w_{z, i j}$. Wind field information is assumed to be available a priori. Figure 1 shows a sample grid where the set of nodes are generated randomly. The black dot is the goal node and the white dots are randomly placed nodes to discretize the environment. The line segment joining two nodes are the edges. Wind information is known at each node. Thus for a transition between $i^{t h}$ node to $j^{\text {th }}$ node the average value of the wind vector at each of the nodes is used. Here the energy required to complete a transition forms a major part of the cost 
function of a transition. Note that the energy $e_{i j}$ required to complete a transition from node $i$ to node $j$ is not necessarily the same as $e_{j i}$. Further, in some cases energy can be harvested along a transition (e.g. when flying through an updraft), leading to a negative transition cost. Dijkstra's algorithm is thus not applicable here. Further, negative cycles are likely to exist (e.g. when the aircraft flies repeatedly through an updraft, gaining energy with each cycle). Hence the Bellman-Ford algorithm cannot be directly applied.

In steady state powered flight, the energy $e_{i j}$ required to fly from node $i$ to $j$ is a function of the wind vector $\mathbf{w}_{i j}$, air speed $v_{a}$ and throttle setting $T$. The heading $\psi_{i j}$ required to fly along the desired ground track between the two nodes is a function of the horizontal component of the wind field and the air speed. Section III summarizes the problem of computing $v_{a}$ which minimizes the energy required for transition $i j$.

It is assumed that the vehicle is in a trimmed, steady state condition during each transition over an edge $i j$, and the time required to change from one trim condition to the next as a node is passed is short compared with the length of time required to complete a transition.

Higher node density can be used in regions where spatial gradients in wind field is large allowing higher resolution trajectories wherever needed. This can be done as apart from the the constraint that the time required to complete a transition at a particular trim condition is long compared with the time required to change trim conditions from one transition to the next, node placement is arbitrary.

In this paper $A^{*}$ is used to plan minimum cost paths to goal. In graph based terms the process in which the search process expands is twofold: (1) selecting the next node to visit and (2) and planning the best path through the rest of the graph to arrive at the goal. The cost function is a linear combination of two terms: $g(n)$, the cost of best found path so far; and $h(n)$, a heuristic function which is an estimate of cost from node $n$ to the goal. Here $h$ is defined as the straight line distance between the current node and the goal, and thus is an estimate of the time required to reach the goal.

The search procedure in this case expands node in order increasing expected cost $f(n)$ which is a linear combination of the two terms:

$$
f(n)=\alpha g(n)+(1-\alpha) h(n)
$$

The function $g(n)$ is evaluated as the total energy required to reach node $n$ :

$$
g(n)=c_{i j}
$$

where $c_{i j}$ is the cost of the transition $i j$ which reaches node $n$, and is defined in Section III.

Thus changing the weight $\alpha$ allows changing the cost function to favor energy gain $(\alpha=1)$ or time to goal $(\alpha=0)$. The effect of varying $\alpha$ on the initial energy can be evaluated by computing the energy required to fly the path generated using a particular value of $\alpha$. Finding the value of $\alpha$ is critical in this context, as both on initial starting energy and time to goal are important parameters governing the utility of a flight path.

\section{Energetics of Soaring Flight}

It is assumed that an on-board controller is able to follow heading, airspeed and throttle commands. Moreover, it is assumed that response to step changes in commands is very fast compared with the duration of a particular command. Hence a point mass model is sufficient to describe vehicle motion for planning purposes (Figure 2). Vehicle kinematics are given by

$$
\begin{aligned}
\dot{x} & =v_{a} \cos \gamma \cos \psi+w_{x} \\
\dot{y} & =v_{a} \cos \gamma \sin \psi+w_{y} \\
\dot{z} & =v_{a} \sin \gamma+w_{z}
\end{aligned}
$$

where $v_{a}$ is airspeed, $\gamma$ is flight path angle with respect to the surrounding airmass, $\psi$ is heading and $w_{x}$, $w_{y}$ and $w_{z}$ are the three components of the $3 \mathrm{D}$ wind vector.

The flight path angle $\gamma$ is a function of airspeed $v_{a}$ and throttle setting $T$, and can be obtained for steady flight. From (Figure 3) resolving forces parallel and perpendicular to the flight path,

$$
\begin{aligned}
& m g \cos \gamma=L+T \sin \alpha \\
& m g \sin \gamma=D-T \cos \alpha
\end{aligned}
$$




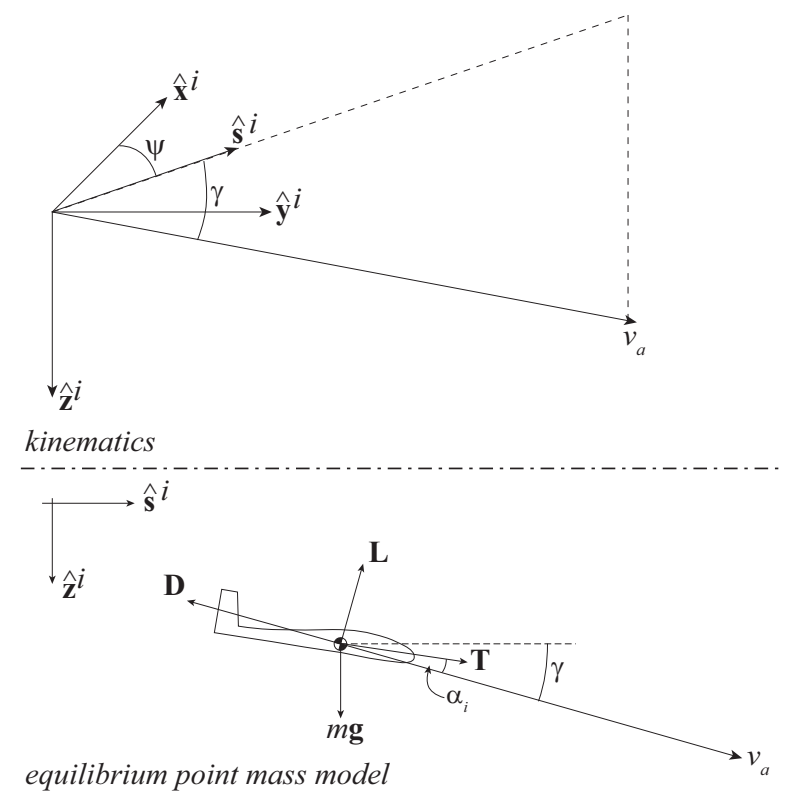

Figure 2. Coordinate frames for vehicle kinematics and equilibrium point mass model for steady trimmed flight.

where $m$ is mass of the vehicle and $\alpha$ is the angle of attack (NB this implicitly assumes that the thrust axis is aligned with the aircraft's body- $x$ axis). Using the standard definition of force coefficients,

$$
\begin{aligned}
& \cos \gamma=\frac{q S}{m g}\left(C_{L}+C_{T} \sin \alpha\right) \\
& \sin \gamma=\frac{q S}{m g}\left(C_{D}-C_{T} \cos \alpha\right)
\end{aligned}
$$

It is assumed that the flight path angle $\gamma$ is small, hence $\sin \gamma \approx \gamma$ and $\cos \gamma \approx 1$. During trimmed cruise flight angle of attack is generally small $\left(3^{\circ}\right.$ to $\left.6^{\circ}\right)$ and thrust is significantly smaller in magnitude than lift. Hence it is further assumed that $C_{T} \sin \alpha$ is negligible compared with $C_{L}$. From Equation 8

$$
C_{L}=\frac{m g}{q S}=\frac{2 m g}{\rho v_{a}^{2} S}
$$

Here $C_{L}$ is lift coefficient, $\rho$ is density of the air, and $S$ is wing area. A polynomial approximation is used for the aircraft's drag polar:

$$
C_{D}=\sum_{i=0}^{n} a_{i} C_{L}^{i}
$$

Typically a second order polynomial is used to represent drag coefficient. However, this is often only valid over a fairly narrow speed range, and here a fourth order polynomial is used.

Substituting into Equation 9, the air mass relative flight path angle for a particular speed and thrust can thus be computed as

$$
\sin \gamma=\frac{q S}{m g}\left(\sum_{i=0}^{n} a_{i} C_{L}^{i}-C_{T}\right)
$$

Combining Equation 10 with Equation 12 and vehicle kinematics, the vehicle's flight path is completely specified by inputs $\mathbf{u}=\left[\begin{array}{lll}v_{a} & \psi & C_{T}\end{array}\right]^{T}$ and wind speed $\mathbf{w}$. This model is adequate as long as the length of time of each trajectory segment is large compared with the time constant of the vehicle's step response with respect to the inputs $\mathbf{u}$.

The steady state kinematics can now be used to analyze flight along a path segment (or edge) between two nodes in the environment. 


\section{A. Flight between two nodes}

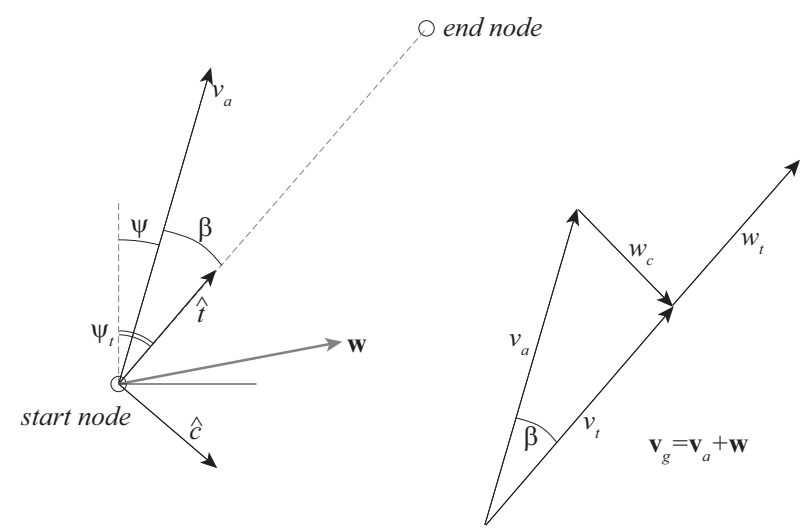

Figure 3. Track coordinate frames (left) and resolution of airspeed and wind vectors into the track coordinate frame (right).

Here the kinematics to fly between two nodes is defined. The line segment joining two successive nodes is the desired ground track. The velocity of the vehicle is decomposed into in-track $v_{t}$ and cross track $v_{c}$ components, hence to maintain flight along the desired ground track $v_{c}=0$. The wind vector is also decomposed into in-track $w_{t}$ and cross-track $w_{c}$ components. $v_{g}$ is the ground speed of the vehicle (Figure 3 ).

From Figure 3 the relationship between air speed, ground speed, heading and ground track for an arbitrary horizontal component of wind can be obtained:

$$
\begin{aligned}
v_{t} & =\sqrt{v_{a}^{2} \cos ^{2} \gamma-w_{c}^{2}} \\
v_{g} & =v_{t}+w_{t} \\
v_{a} \cos \gamma \sin \beta & =w_{c}
\end{aligned}
$$

where $v_{g}$ is the magnitude of the ground speed, $v_{a} \cos \gamma$ is the projection of the airspeed vector onto the horizontal plane and $\beta$ is the angle between the airspeed vector and the desired ground track. Recall that flight path angle $\gamma$ is assumed to be small, hence $\cos \gamma \approx 1$ and the ground speed is $v_{g} \approx \sqrt{v_{a}^{2}-w_{c}^{2}}+w_{t}$. The constraint $v_{c}=0$ is expressed in Equation 15.

The aircraft heading to maintain the desired ground track is $\psi=\psi_{t}-\beta$. Hence

$$
\psi=\psi_{t}-\sin ^{-1} \frac{w_{c}}{v_{a}}
$$

Clearly heading $\psi$ is dependent on airspeed $v_{a}$. The problem now is to determine the optimal value of airspeed $v_{a}$ and thrust coefficient $C_{T}$ for flight between two nodes.

\section{B. Minimizing Energy Loss}

Using the kinematics defined earlier the steady state airspeed which minimizes the energy lost over a segment (or equivalently, maximizes the energy gained) will be determined. Total energy is

$$
E_{t o t}=m g h+\frac{m}{2} v_{a}^{2}+E_{s}
$$

where $h$ is altitude and $E_{s}$ is on-board stored energy. Specific total energy is

$$
\begin{aligned}
e_{t o t}=\frac{E_{t o t}}{m g} & =h+\frac{v_{a}^{2}}{2 g}+\frac{E_{s}}{m g} \\
& =h+\frac{v_{a}^{2}}{2 g}+e_{s}
\end{aligned}
$$

Minimizing energy lost over a segment means maximizing $\frac{\Delta e_{\text {tot }}}{\Delta s}$. In steady state flight this is equivalent to maximizing $\frac{\dot{e}}{v_{g}}$, in other words flying to maximize range. The rate of change of specific energy is

$$
\dot{e}_{t o t}=\dot{h}+\frac{v_{a} \dot{v}_{a}}{g}+\dot{e}_{s}
$$

$$
5 \text { of } 18
$$


In steady flight acceleration is zero, hence

$$
\dot{e}_{t o t}=\dot{h}+\dot{e}_{s}=-\dot{z}+\dot{e}_{s}
$$

Recall that $z$ is positive down and $\dot{z}$ is defined in Equation 5 .

The quantity $\dot{e}_{s}$ is the rate of change of on-board stored energy. This is dependent on motor power and the efficiency of energy conversion.

$$
\dot{e}_{s}=-\frac{T v_{a}}{m g \eta_{e c} \eta_{p}}=-\frac{q S}{m g} \frac{C_{T} v_{a}}{\eta_{e c} \eta_{p}}
$$

where $\eta_{e c}$ is the net efficiency of energy conversion from source to shaft (in electrical power systems this is the product of motor efficiency and speed controller efficiency $\eta_{e c}=\eta_{e s c} \eta_{m}$ ) and $\eta_{p}$ is the propeller efficiency.

Thus the rate of change of total energy (in steady state flight) is

$$
\dot{e}_{t o t}=-\left(v_{a} \sin \gamma+w_{z}\right)-\frac{q S}{m g} \frac{C_{T} v_{a}}{\eta_{e c} \eta_{p}}
$$

and maximum range flight occurs when one maximizes

$$
\frac{\dot{e}_{t o t}}{v_{g}}=-\frac{v_{a} \sin \gamma+w_{z}}{\sqrt{v_{a}^{2}-w_{c}^{2}}+w_{t}}-\frac{q S}{m g} \frac{C_{T} v_{a}}{\eta_{e c} \eta_{p}\left(\sqrt{v_{a}^{2}-w_{c}^{2}}+w_{t}\right)}
$$

Many missions include altitude restrictions (for example to ensure separation or to ensure appropriate sensor coverage). Hence aircraft motion will be restricted to constant altitude. This has additional benefit of reducing graph size, improving computational feasibility. At constant altitude $\dot{h}=0$. Hence $v_{a} \sin \gamma=-w_{z}$ :

$$
\sin \gamma=\frac{q S}{m g}\left(\sum_{i=0}^{n} a_{i} C_{L}^{i}-C_{T}\right)=-\frac{w_{z}}{v_{a}}
$$

Hence the problem is to find the airspeed va and throttle setting $C_{T}$ which solves the optimization problem

$$
\begin{aligned}
\text { maximize } & \frac{\dot{e}_{s}}{v_{g}} \\
\text { subject to } & \frac{q S}{m g}\left(\sum_{i=0}^{n} a_{i} C_{L}^{i}-C_{T}\right)=-\frac{w_{z}}{v_{a}} \\
& v_{g}=\sqrt{v_{a}^{2}-w_{c}^{2}}+w_{t} \\
& v_{a, \text { min }} \leq v_{a} \leq v_{a, \text { max }} \\
& C_{T, \text { min }} \leq C_{T} \leq C_{T, \text { max }} \\
& v_{g}>0
\end{aligned}
$$

The constraint defined by Equation 26 limits the vehicle to constant altitude flight. Equation 27 and Equation 30 together ensure that the vehicle always proceeds forward along the desired ground track. Airspeed limits are defined by Equation 28 and thrust coefficient limits are defined by Equation 29

\section{Regenerative Soaring}

For battery powered aircraft a wind milling propeller (or a ram air turbine) can be used to recharge batteries at the cost of increased drag. One can thus trade potential energy (altitude) for stored electrical energy. When flying through a strong enough updraft it is possible to either: (1) gain potential energy by climbing at constant speed; (2) gain kinetic energy by flying at higher speed but at constant altitude; (3) gain stored electrical energy by flying at constant speed and altitude and windmilling the propeller. Of course a combination of the three can also occur.

Using a windmilling propeller to gain electrical energy is known as regenerative soaring, and was first described by MacCready. ${ }^{19}$ 
In the context of the optimization problem posed above, setting $C_{T, \min }=0$ means that regeneration cannot occur (as $\dot{e}_{s}$, the energy expended from the on-board supply, has a minimum value of zero). Permitting negative values of $C_{T, \min }$ implies that $\dot{e}_{s}$ can be positive quantity (Equation 21), and thus the energy (potential and/or kinetic) can be transferred to batteries.

For constant altitude flight $\dot{e}_{s}$ signifies cost of transition between two nodes. Energy expenditure $\dot{e}_{s}$ will in general be positive, i.e, energy is required to make a certain transition. Thus $\dot{e}_{s}<0$ means negative energy expenditure or gaining energy from the atmosphere which can be stored by charging the batteries. There is a limit to the amount to which the batteries can be charged. Thus regenerative soaring is allowed up to the full capacity of the batteries and once the batteries have been fully charged $C_{T, \min }$ is set to zero.

\section{Cost Of Transition between Nodes}

Since $e$ has dimension distance, $\frac{\dot{e}}{v_{g}}$ is a dimensionless quantity. Equation 10 and Equation 12 together show that flight path angle with respect to air is a function of airspeed and throttle setting (because in trimmed flight $C_{L}$ is a function of airspeed). Hence for flight paths $\frac{\dot{e}}{v_{g}}$ is a function only of airspeed and thrust coefficient, and the airspeed and thrust coefficient which maximizes energy gained over a segment can be computed. Note that in zero wind the energy change is always negative.

The cost of a transition is defined as

$$
c_{i j}=-\frac{\dot{e}}{v_{g}}
$$

i.e. the energy expended in a transition. A generic function minimizer (such as MatLab's fmincon) can be used to find $v_{a}^{\text {opt }}$ and $C_{T, \text { optimal }}$ which minimizes $c_{i j}$ while ensuring that constraints such as airspeed limits (stall and maximum speed) are not exceeded. Once $v_{a}^{o p t}$ has been computed the required heading to maintain the desired ground track between the start and end nodes is computed from Equation 16.

The procedure outlined above is used to find the minimum energy loss $c_{i j}^{*}$, optimal airspeed $v_{a}^{\text {opt }}$, optimal thrust coefficient $C_{T}^{o p t}$ and required heading to fly the desired ground track for each of the allowable transitions in the environment. A graph based path planner such as $A^{*}$ can now be used to compute a path to the goal which balances energy expenditure and another cost such as time or distance to goal.

\section{Weighing energy gain vs. distance to goal}

To find the critical value of $\alpha$ is the key to use $\mathrm{A}^{*}$ in this application. The value of $\alpha$ is important tradeoff between energy gained and time to reach the goal. If the value of $\alpha$ is too large the aircraft will will spend too much time looking for energy and not go to the goal quickly enough and if the value of $\alpha$ is too small it wont go easily to the easily exploitable energy sources.

The energy map approach described earlier ${ }^{18}$ is used as a comparison to evaluate the utility of paths generated using $\mathrm{A}^{*}$ approach. The energy map is an upper bound on the minimum energy required to reach the goal in a given wind field. Thus the initial starting energy when compared with the energy map will give us an intuitive understanding of the critical value of $\alpha$ to choose to successfully use $\mathrm{A}^{*}$ for soaring application.

Two example wind fields are used to examine the effect of the weight $\alpha$ on flight paths: a thermal-like field consisting of two regions of upwards moving air and two regions of downwards moving air and a ridge wind field consisting of two parallel hemi-cylindrical ridges. For both cases a rectangular Cartesian grid is used to discretize the environment. Allowable transitions can occur to nearest neighbors along the sides and diagonals of the grid.

Calculations were performed for an RnR Products SB-XC glider; parameters are given in the Appendix. The SB-XC glider is an electrically powered aircraft. Regenerative soaring is allowed in these simulations. The battery pack is assumed to be a 4S1P (4 series, 1 parallel) lithium-polymer battery with total capacity of $4.9 \mathrm{Ah}$. Pack voltage is $14.8 \mathrm{~V}$, resulting in a total capacity of $261 \mathrm{~kJ}$. At a vehicle mass of $10 \mathrm{~kg}$ the specific energy of the pack is $2748 \mathrm{~m}$. For simulations presented here this is reduced to $2500 \mathrm{~m}$. Thus for regenerative soaring the total energy expenditure is permitted to reach a minimum of $-2500 \mathrm{~m}$. Positive values of energy expenditure thus represent energy lost and negative values of energy expenditure represent energy gained from the atmosphere. 


\section{A. Thermal-like wind fields}

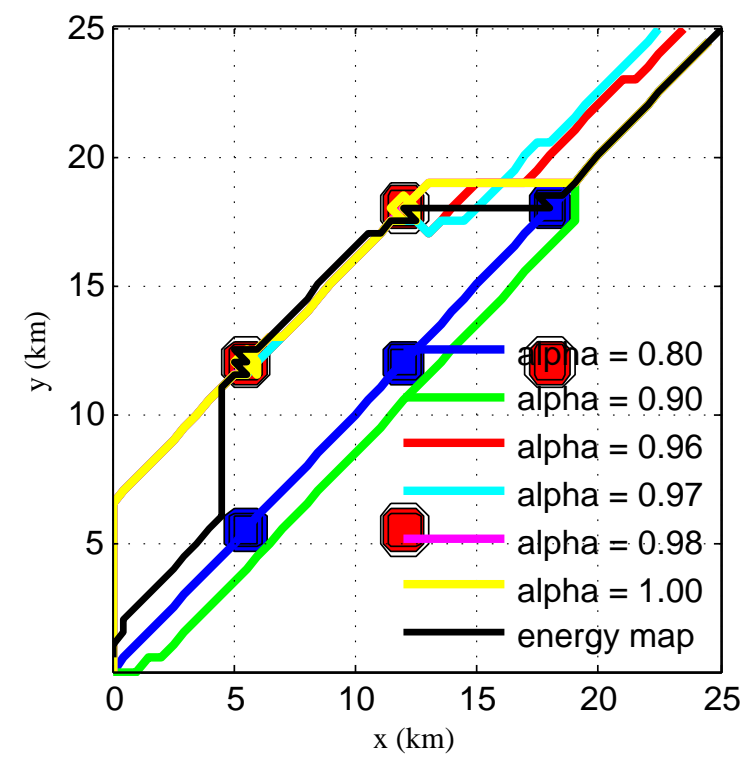

(a) $\mathrm{A}^{*}$ paths for thermal-like wind field

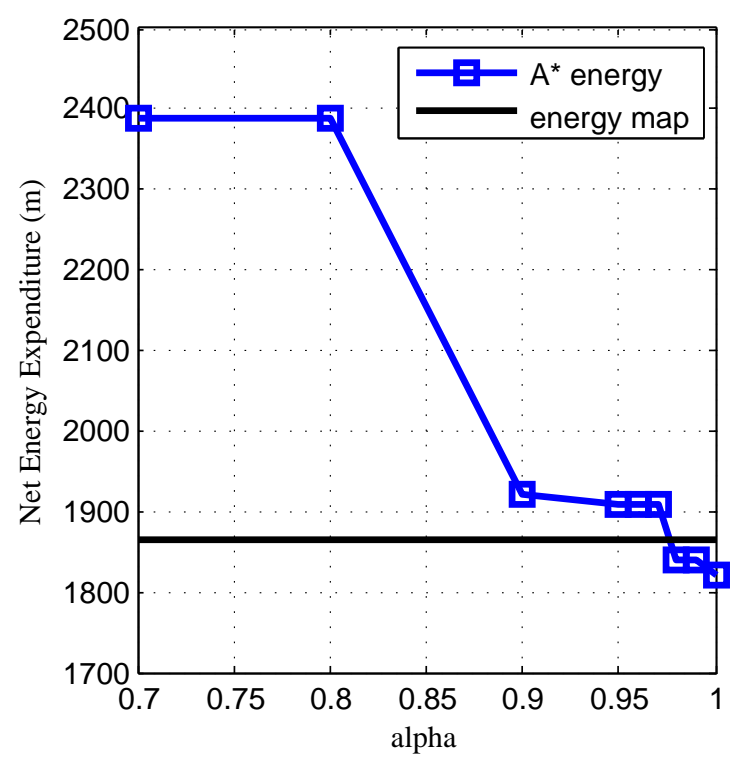

(b) Variation of required initial energy with $\alpha$

Figure 4. Subfigure (a): A* and energy map paths to the goal; Subfigure (b): Comparison of Energy expended to reach the goal.

Consider a square region of $25 \mathrm{kms} \mathrm{X} 25 \mathrm{kms}$ which is divided in uniform cartesian grid of $50 \mathrm{X} 50$ nodes which has different regions of vertical wind (both upwards and downward wind). Upward moving air representative of thermal of approximate radius of $0.5 \mathrm{Km}$ are located at $\mathrm{x}=12.5 \mathrm{~km}$ and $\mathrm{y}=6.25 \mathrm{~km}$, $\mathrm{x}=18.75 \mathrm{~km}$ and $\mathrm{y}=12.5 \mathrm{~km}, \mathrm{x}=6.25 \mathrm{~km}$ and $\mathrm{y}=12.5 \mathrm{~km}$ and $\mathrm{x}=12.5 \mathrm{~km}$ and $\mathrm{y}=18.75 \mathrm{~km}$. Similar regions of downward moving air are located at $\mathrm{x}=6.25 \mathrm{~km}$ and $\mathrm{y}=6.25 \mathrm{~km}, \mathrm{x}=12.5 \mathrm{~km}$ and $\mathrm{y}=12.5 \mathrm{~km}$ and $\mathrm{x}=18.75 \mathrm{~km}$ and $\mathrm{y}=18.75 \mathrm{~km}$. In Figure 4a upward moving air is in the regions shown in red and downward moving air in regions shown in blue. The goal is the origin of the co-ordinate system.

The sample wind field is so chosen as it has both regions where the aircraft can gain energy and lose energy. A "good" path planner should avoid regions where it loses energy but should try to fly in proximity of those regions where it can gain energy. This example also shows the effect of discretization on the final solution.

Figure 4a shows $\mathrm{A}^{*}$ paths to goal from a distant point of $(x, y)=(25 \mathrm{~km}, 25 \mathrm{~km})$. As the weight factor $\alpha$ is varied between zero and one, the path planned for the UAV shows clear changes. When $\alpha$ equals zero there is no weight on the energy available from the atmosphere and the cost function is driven by only the distance to goal. In this case all paths with $\alpha<0.8$ behave similarly, and the optimal path is straight to the goal, thus the aircraft flies straight through the energetically unfavorable regions of downwards moving air. The blue path in Figure 4(a) shows that this is indeed followed. When $\alpha=0.9$ the path avoids the regions of downwards moving air (green path) but does not divert further to exploit the energy in the other rising pockets.

As the value of $\alpha$ is increased further $(\alpha>0.96)$ paths begin to exploit upwards moving air. More time is spent in the region of upwards moving air as more emphasis is placed on energy gain. When $\alpha=1$ time to goal is irrelevant, and the flight path traverses every node where energy gain is possible. Note that the path still does not crosses to other nodes where there are unexplored regions of upward moving air. This is because of the unfavorable wind along the diagonal which does not not allow search procedure to advance in those directions and goal is reached by the planner before all the nodes are traversed. The path in black shows the energy-map computed path. ${ }^{18}$

For each value of $\alpha$ the energy expended along the path is calculated. As seen from Figure $4 \mathrm{~b}$ the energy expended for $\alpha=1$ is much lower compared to the other values. Energy expended for values of $\alpha \leq 0.8$ is high as the paths goes straight through the regions of downward moving air. Energy expended is considerably 
reduced for $\alpha \geq 0.9$ as the aircraft starts avoiding the unfavorable wind. But the real jump in the energy expended is found at $\alpha=0.96$. For values of $\alpha>0.96$ the energy expended along the path is lower than that expended if followed the energy map path.

Comparing the energy expended for each path shows the influence of the distance:energy weight on the computed paths. Further, comparison with the energy map path shows that for $0.96<\alpha<0.97$ the required initial energies are roughly equal. Higher weights on energy reduce required initial energy but the cost is significantly longer paths to goal. Similar results were found for planning scenarios using ridge-like wind fields.

\section{B. Orographic Wind Fields}

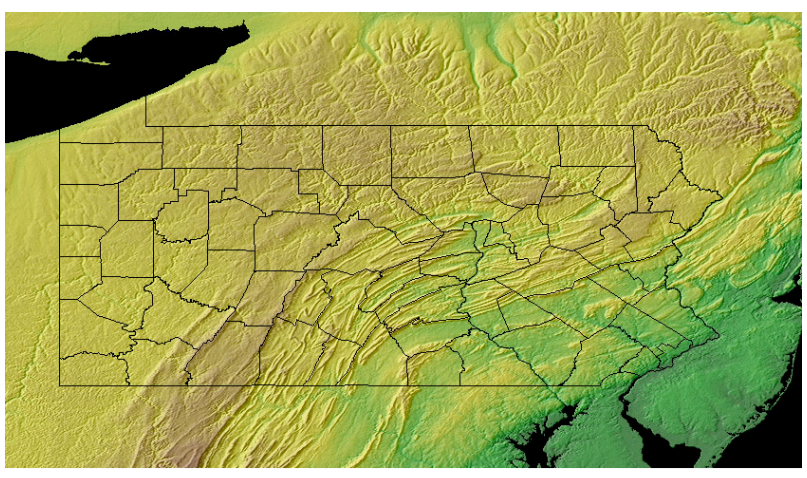

(a) Digital elevation map of Pennsylvania

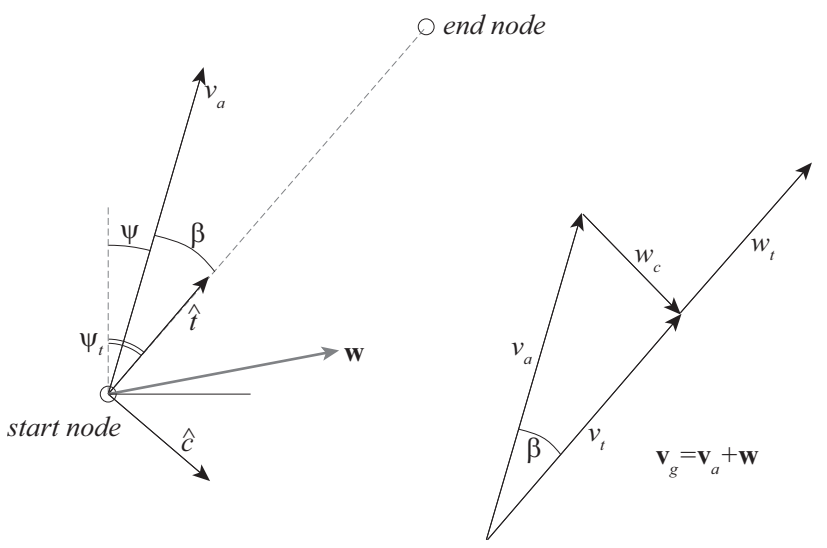

(b) Cross section of parallel ridges and wind field

Figure 5. Subfigure (a): Topography of central Pennsylvania showing Appalachian mountains; Subfigure (b): Schematic of ridges and potential flow solution of wind field.

Orographic lift occurs when wind is deflected upwards by terrain, for example along coastlines with onshore breezes or along hills and ridges. Here the case of flight along and across ridges is considered. For illustrative purposes terrain representative of the Appalachian Mountains of Central Pennsylvania is used in these simulations. This is done both because this is representative of real terrain (Figure 5) and because it permits flight in regions of upwards, downwards and horizontal moving air. A distance of $12 \mathrm{~km}$ separates two parallel ridges. A global coordinate frame is defined with y parallel to the ridges, so that the ridge centerlines are located at $\mathrm{x}=4 \mathrm{~km}$ and $\mathrm{x}=16 \mathrm{~km}$. Each ridge is modeled as an infinitely long hemi-cylinder with radius of $300 \mathrm{~m}$, to compute the wind field potential flow method is used. Potential flow cannot model flow separation on the downwind side of the ridges. But one can find upward air motion on the upwind side. This upwind side is the favorable side where one can expect the aircraft to gain energy. Intuition suggests that the aircraft will tend to follow the upwind sides of ridges, thus the flow on the downwind sides of ridges is less critical to trajectory planning (except for the times when the vehicle must traverse these non-favorable regions).

The origin is at $(0 ; 0)$ and the terrain is over an area defined by $0 \leq x \leq 20 \mathrm{~km}$ and $0 \leq y \leq 100 \mathrm{~km}$. Here constant altitude flight at $310 \mathrm{~m}$ (just enough to clear the ridges) is considered. In this case grid spacing is not uniform: rather nodes are more densely packed along areas where the wind field changes rapidly, allowing more accurate paths without greatly increasing computation requirement. For this example a non uniform Cartesian grid is used, with finer grid spacing over the ridges, where the wind field changes rapidly over short distances. And wider grid spacing is used between the ridges where the wind is roughly constant. Figure 5b shows a vector plot of the computed wind field at the $\mathrm{x}$ coordinates of the grid. Spacing varies from a minimum of $100 \mathrm{~m}$ to a maximum of $1000 \mathrm{~m}$. Grid spacing in the y direction is constant at $1000 \mathrm{~m}$.

Simulation results were carried out with $w_{x, \infty}=-5 \mathrm{~m} / \mathrm{s}$ (which results in maximum vertical component of wind of approximately $3.0 \mathrm{~m} / \mathrm{s}$ along the ridge). The wind blows from right to left. Thus there is upward moving air (which permits energy extraction) on the right side (positive $x$ ) of the ridges. Flight paths were computed for four different starting positions: $(x, y)=(20 \mathrm{~km}, 100 \mathrm{~km}),(x, y)=(20 \mathrm{~km}, 50 \mathrm{~km})$, $(x, y)=(20 \mathrm{~km}, 20 \mathrm{~km})$, and finally $(x, y)=(10 \mathrm{~km}, 70 \mathrm{~km})$. Flight paths were generated for each starting position for varying values of $\alpha$ and results are compared with flight path computed using the energy map. 


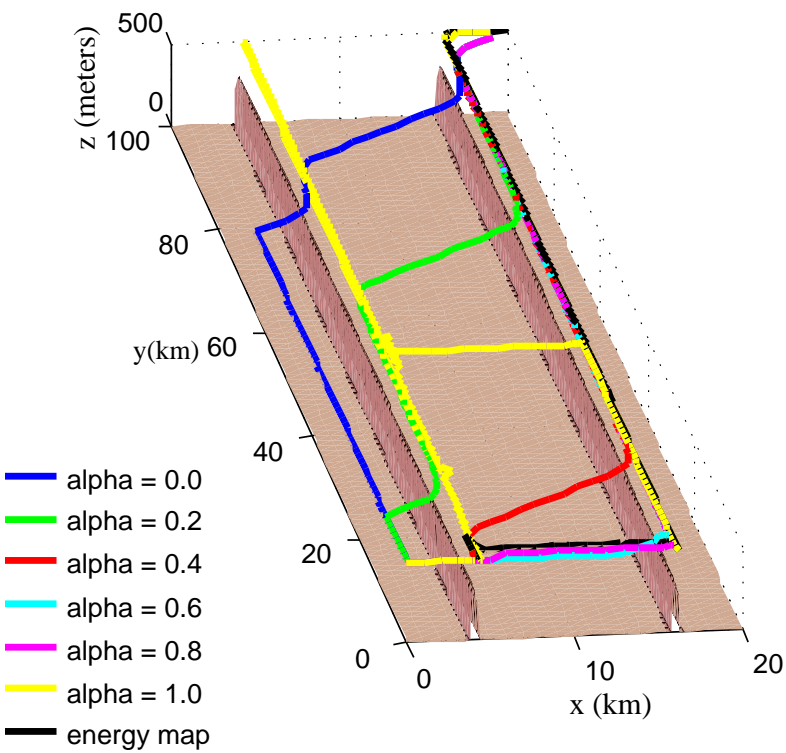

(a) Comparison of $A^{*}$ paths and Energy Map path

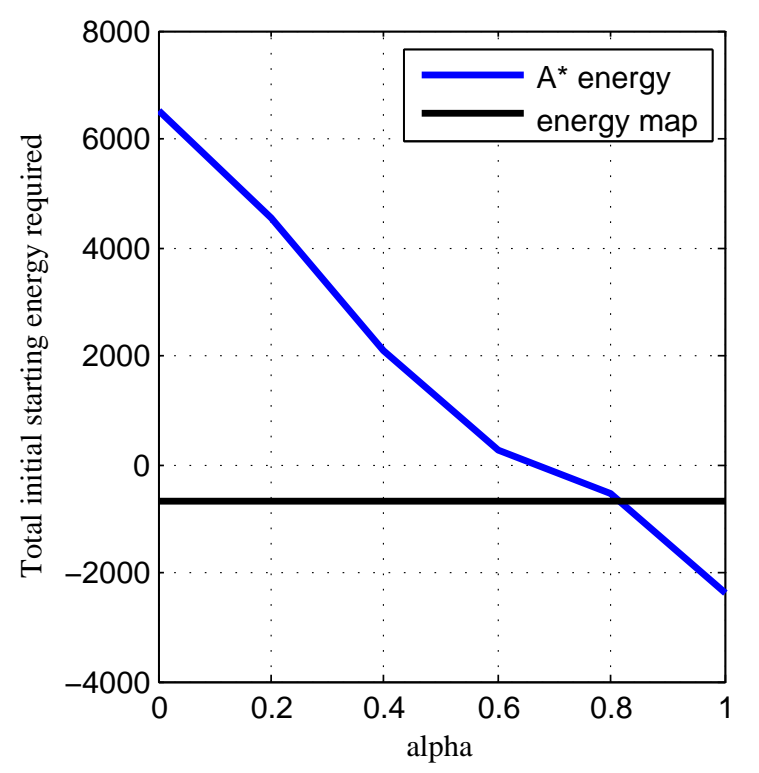

(b) Energy expenditure for varying $\alpha$ and the energy map.

Figure 6. Left: Different paths to the goal starting from $x=20 \mathrm{~km}$ and $y=100 \mathrm{~km}$; Right: Comparison of the initial starting energy for $x=20 \mathrm{~km}$ and $y=100 \mathrm{~km}$

Here results from only one of the starting points, namely $(x, y)=(20 \mathrm{~km}, 100 \mathrm{~km})$ are shown. Starting points from the other regions also produced similar results.

From Equation 1 it is evident that for $\alpha=0$ the energy required is not included in the total cost function, thus the flight path goes straight towards the goal irrespective of wind field. Note that the path is affected by the grid, which is Cartesian and non-uniform. For $\alpha=1$ only energy gain is considered, and the flight path will follow the upwind side of ridges as much as possible.

Figure 6 a shows the path to the goal from a distant point of $\mathrm{x}=20 \mathrm{~km}$ and $\mathrm{y}=100 \mathrm{~km}$. As $\alpha$ is varied from 0 to 1 flight paths goes more in the regions of upward moving air. For $\alpha=0$ the path goes straight towards the goal. Note the effect of grid on the path. Instead of moving straight towards the goal the vehicle opts for the manhattan distance. As the value of $\alpha$ keeps on increasing the flight paths are seen to wander near regions of the upwind where it can gain energy. The energy map path is also shown in the same figure. For $\alpha=0.8$ almost similar path is seen than that of energy map. The energy map path always moves towards the goal. It leaves the first ridge and moves over to the second ridge in between as those two will have same energy gains and are effectively equivalent paths. The path for $\alpha=1$ is interesting. Flight paths are seen to zigzag in the first ridge and then when all the nodes are exhausted the path crosses over to the next ridge. Upon reaching the next ridge the aircraft continues to gain energy moving to and fro in the upwind side of the ridge and eventually reaches the goal.

Figure $6 \mathrm{~b}$ shows the expended energy for different values of $\alpha$. Results match the intuition that as the importance of energy gain is increased, the net expended energy is reduced. Because the starting point is at the extremum of the task area the $\mathrm{A}^{*}$ path (which would otherwise allow motion away from the goal) is equivalent to the energy map path (which does not).

Figure 7 shows the value of energy, velocity and thrust coefficient along the path for different values of alpha. For $\alpha=0$ as seen from Figure 7a the energy required increases steadily. Also note that with a battery capacity of $2500 \mathrm{~m}$ a flight path straight to the goal is not feasible, thus energy gain from the atmosphere (i.e. soaring flight) is required to reach the goal. There are two places where the energy dips a little bit. These are the regions where the path crosses the ridges. The velocity profile shows an increase in velocity while moving through the downward air in the lee ward side of the mountains, while velocity decreases and thrust coefficient touches negative as the vehicle passes through the regions of upward moving air. For the other cases of $\alpha$ one can see such values of velocity and thrust coffering as the aircraft crosses the ridges. As the value of $\alpha$ increases total energy required decreases. It can be seen from the velocity profiles that 

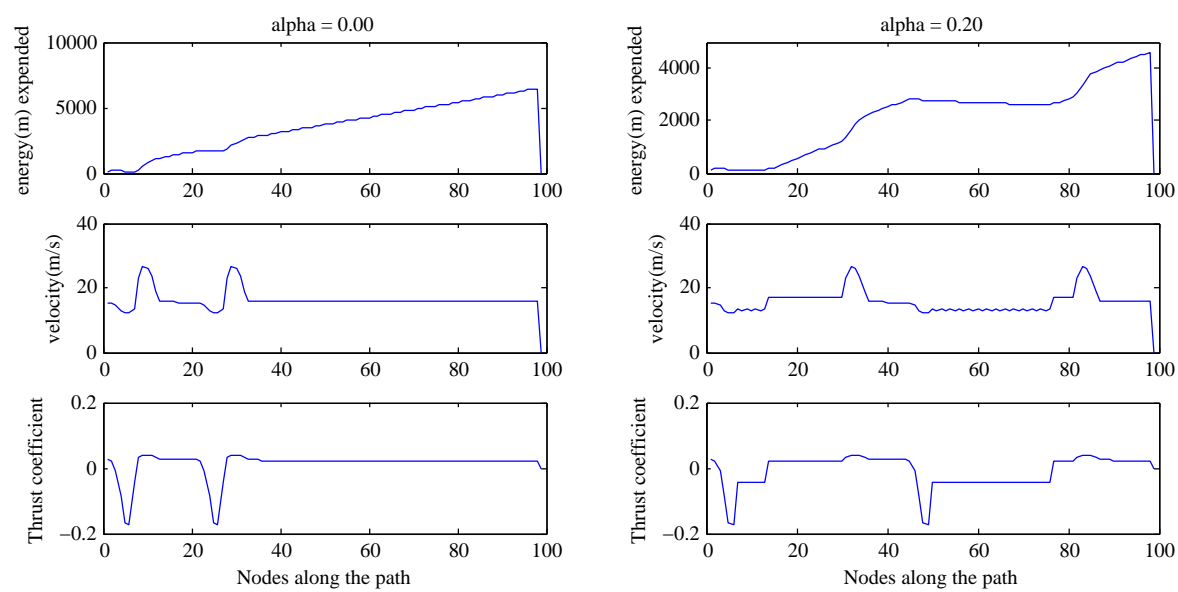

(a)

(b)
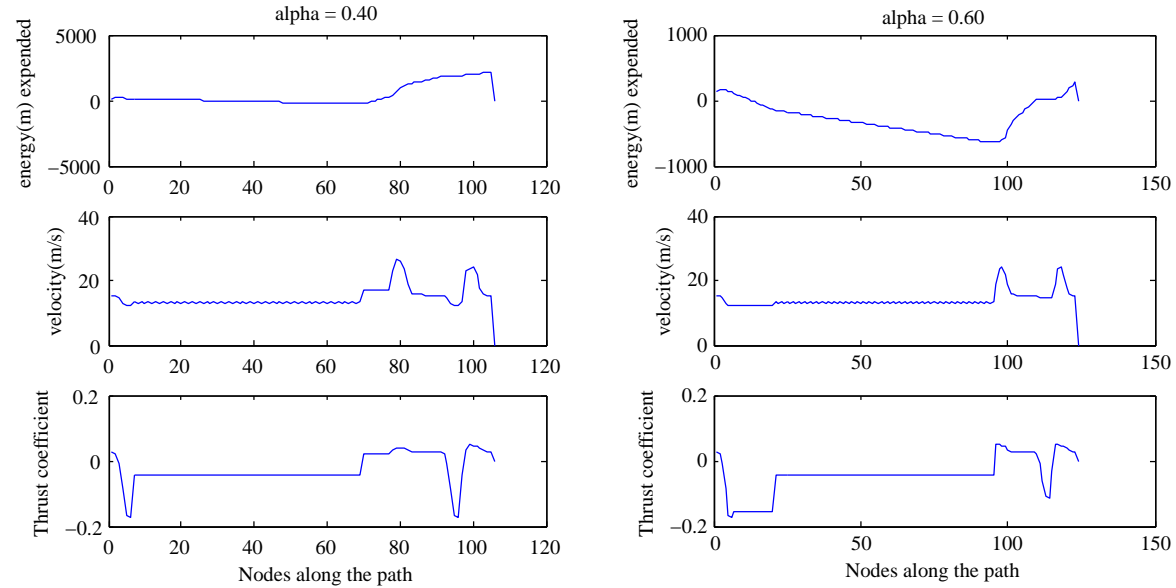

(c)

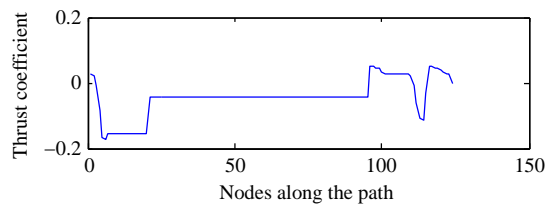

(d)
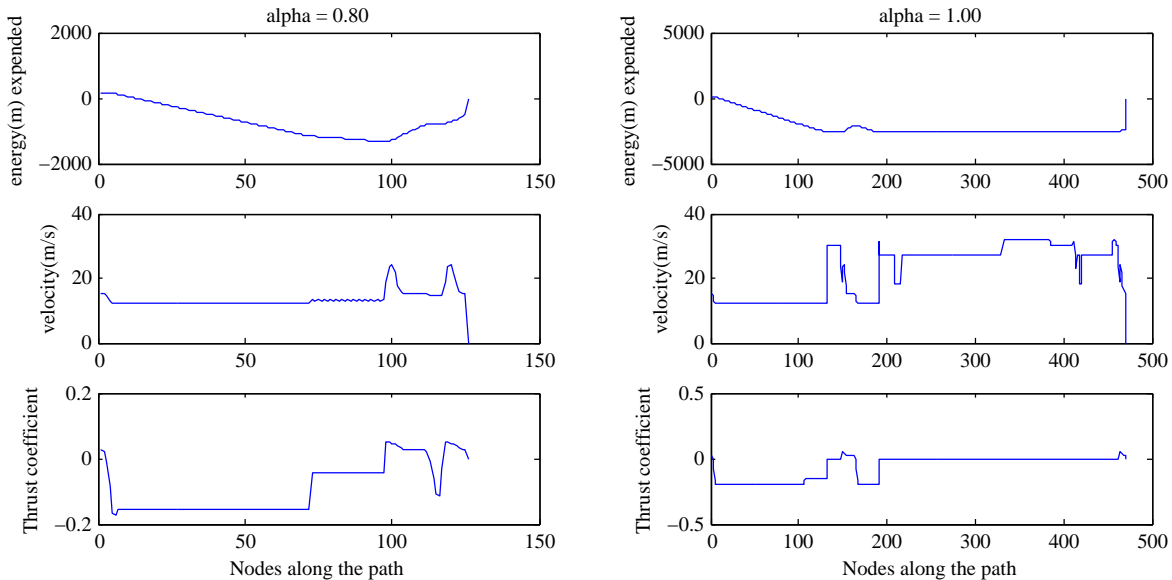

(e)

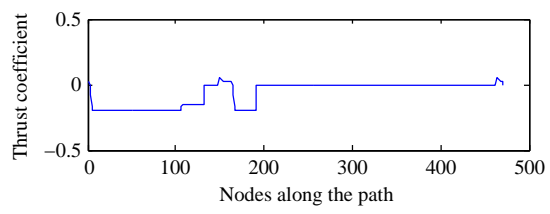

(f)

Figure 7. Energy expended, Velocity and Thrust Coefficient along the paths for different values of $\alpha$. Note the number of nodes along a path for a particular value of $\alpha$ increases as the value of $\alpha$ increases signifying more time to reach the goal. Energy expended along the path is negative when the aircraft gains energy and this increases the stored energy. 


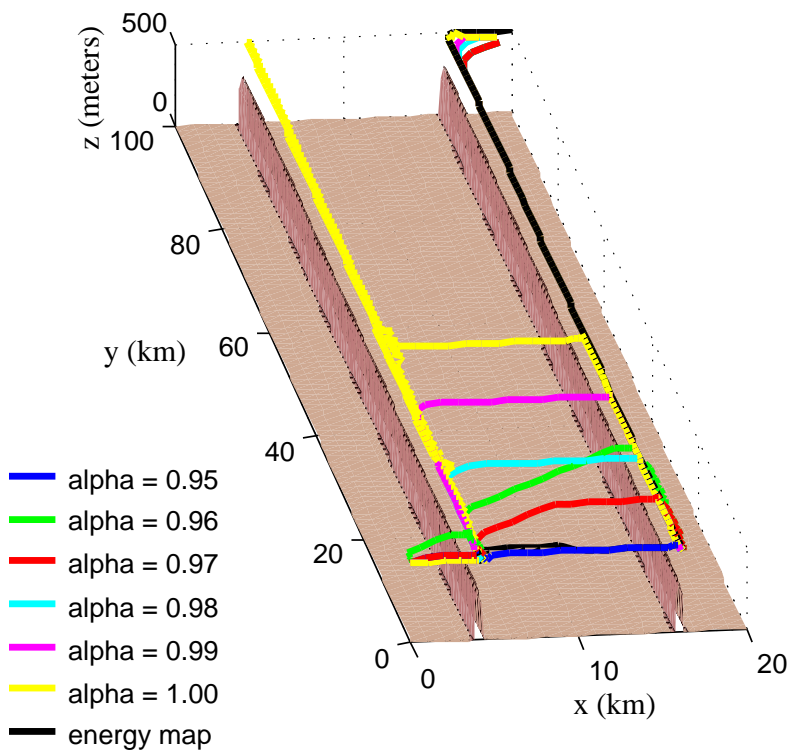

(a) Comparison of $A^{*}$ paths and Energy Map path

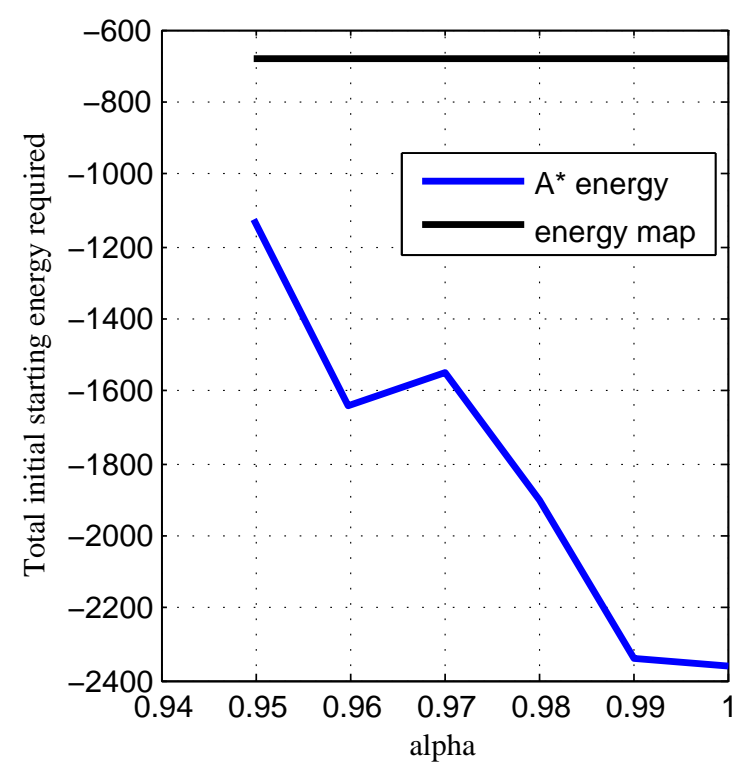

(b) Initial Starting energy for varying $\alpha$ and energy map

Figure 8. Left: Different paths to the goal starting from $x=20 \mathrm{~km}$ and $y=100 \mathrm{~km}$; Right: Comparison of the initial starting energy for $x=20 \mathrm{~km}$ and $y=100 \mathrm{~km}$

the aircraft spends very little time in the leeward side of the mountain where it loses energy. Velocity in this cases hits the peaks. For $\alpha=1$ the energy dips very quickly and hits $-2500 \mathrm{~m}$. This is the point where regenerative soaring is stopped and minimum thrust co-efficient is set to be zero because the batteries are fully charged. Thus velocity shoots up and thrust remains zero. It is at this point the aircraft decides to leave the first ridge. While crossing over to the other ridge the aircraft has to again expend energy and one can see drop in velocity and increase in thrust coefficient. After reaching the second ridge again the vehicle gains energy and hits $-2500 \mathrm{~m}$ of energy and thrust coefficient remains zero for the next rest of the path and also the velocity becomes high.

Thus in these cases one can see a big jump in starting energy required as $\alpha$ is varied from 0 to 1 . Increasing alpha reduces the starting energy required but the time to reach the goal also increases dramatically (as indicated by the number of nodes traversed during the flight: 500 nodes are traversed for $\alpha=1$ while less than 100 nodes are traversed for $\alpha=0$ ). (as indicated by the number of nodes in case of $\alpha=1$ which is almost equal to 500 while compared to less than 100 for $\alpha=0$ ).

Thus to find an effective value of $\alpha$ results with higher values of alpha were analyzed.

Figure 8a shows the paths to the goal as $\alpha$ is varied between 0.95 to 1.0. Close observation of the paths reveal that there is a certain value of alpha which shows a finite jump in the trajectory. For $\alpha=0.96$ the path for the first time goes to and fro through the ridge and then crosses when the distance function takes over. From Figure $8 \mathrm{~b}$ also one can see that there is a sharp decrease in energy required to reach the goal as $\alpha$ is increased from 0.95 to 0.96 . If alpha is increased further there is little improvement in terms of energy required. For $\alpha=1$ the energy reaches the maximum value of maximum allowable charging limit of the batteries.

Similar flight paths are generated four different starting positions: $(x, y)=(20 \mathrm{~km}, 100 \mathrm{~km}),(x, y)=$ $(20 \mathrm{~km}, 50 \mathrm{~km}),(x, y)=(20 \mathrm{~km}, 20 \mathrm{~km})$, and finally $(x, y)=(10 \mathrm{~km}, 70 \mathrm{~km})$. After analyzing the data obtained from all the starting positions the critical value of $\alpha$ for which energy gain can be maximized without redundant paths is found to be $\hat{\alpha}=0.96$.

From Figure 9 shows the energy, velocity and thrust coefficient for the different values of $\alpha . \alpha=0.96$ shows saw tooth nature of the velocity and thrust coefficient. This happens due to zig-zag flying of the aircraft through the upwind side. Thus $\alpha=0.96$ seems to be the transition value of alpha where there is correct correlation between energy gained and distance left to travel. Higher values of alpha leads to redundant paths which increase the time to reach the goal unnecessarily. 

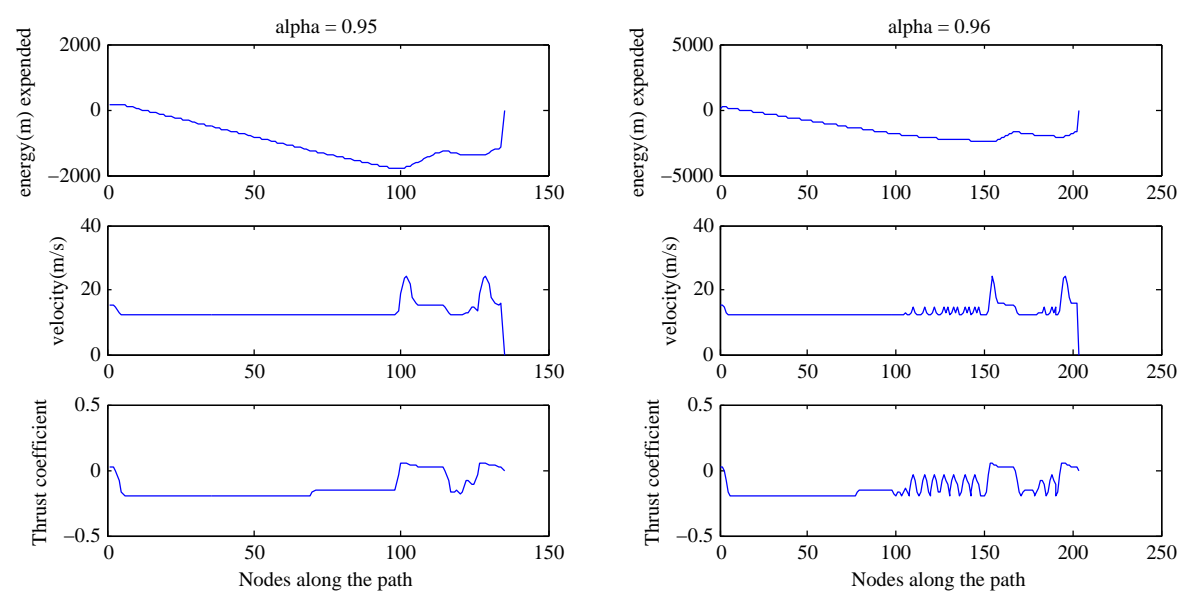

(a)

(b)
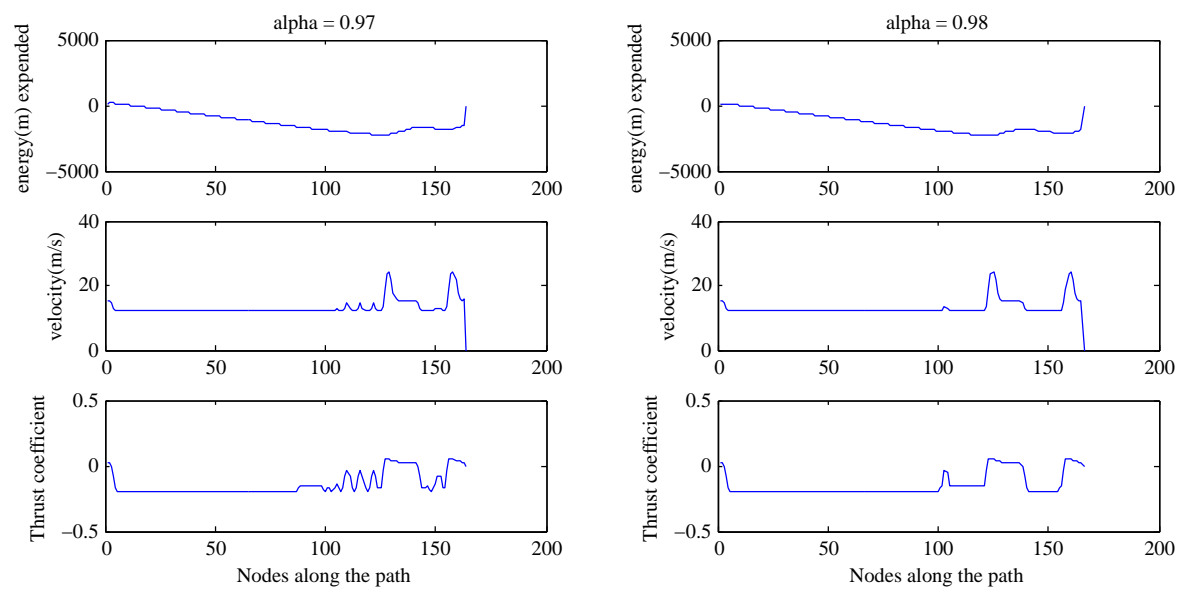

(c)

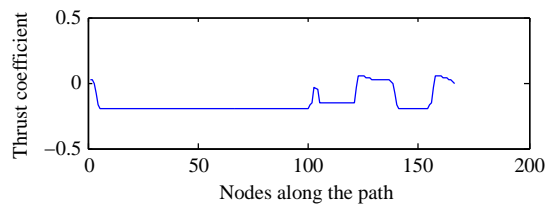

(d)
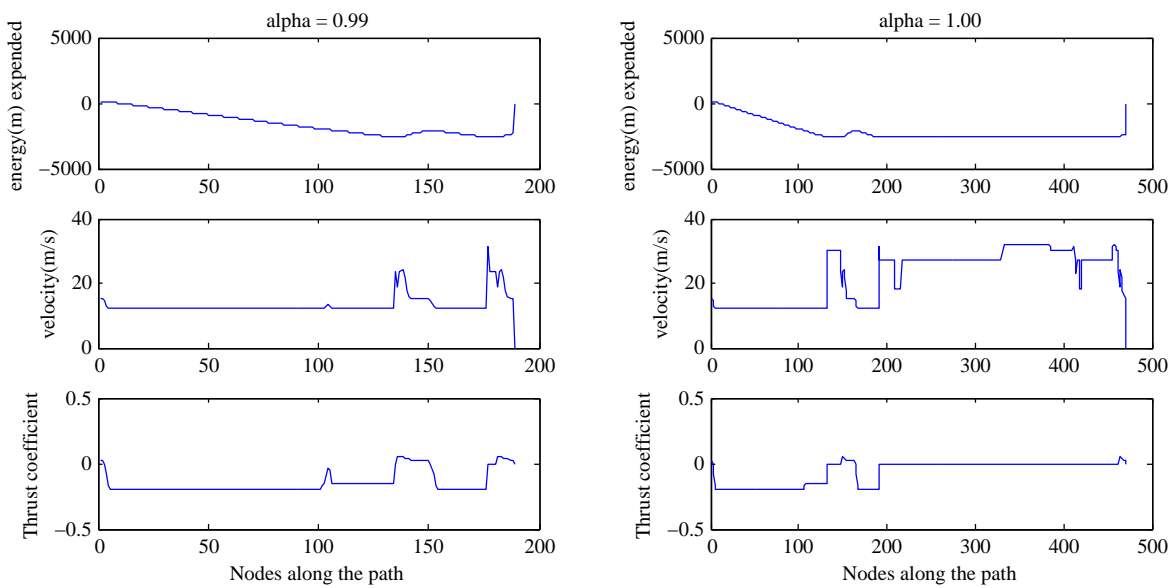

(e)

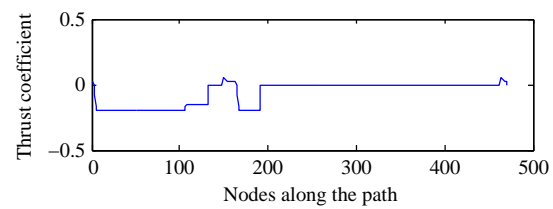

(f)

Figure 9. Energy expended, Velocity and Thrust Coefficient along the paths for different values of $\alpha$. Note the number of nodes along a path for a particular value of $\alpha$ increases as the value of $\alpha$ increases signifying more time to reach the goal. Energy expended along the path is negative when the aircraft gains energy and this increases the stored energy. 
The A* approach which weights energy and time to goal is able to plan trajectories. By changing the relative weight of energy cost vs. distance to goal qualitative statements about flight paths can be made, but the flight paths show a strong jump once $\alpha \geq 0.96$. Once value of energy weight causes the flight path to divert from the goal in favor of gaining energy, flight paths will divert as far as the task area allows, with no means of heading to the goal once "sufficient" energy has been gained. This suggested that the upper limit on "useful" values of energy weight is roughly 0.96 . These simulation results are now applied to see the performance of $\mathrm{A}^{*}$ in a real environment.

\section{Energy Harvesting from Mountain Wave}

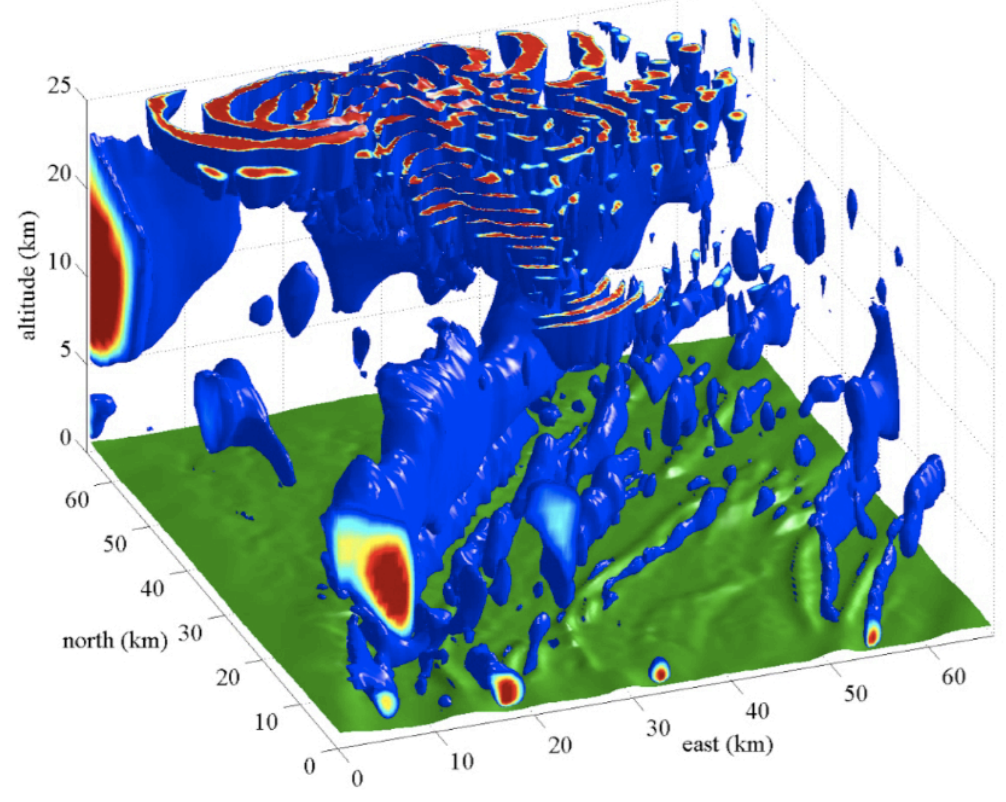

Figure 10. Isosurface Plots of wind field where the vertical speed of air greater than $0.56 \mathrm{~m} / \mathrm{s}$ (minimum sink rate of the aircraft).

The approach is now applied to the problem of computing trajectories for a realistic wind field. Results of a simulation of wind field of mountain wave computed using WRF-ARW (Weather Research and ForecastingAdvanced Research WRF) version 2.2 are used, the wind field's structure is shown in Figure 10 for a point in time. The blue isosurfaces bound regions where energy can be harvested from the atmosphere. In these regions the vertical component of wind is greater than $0.56 \mathrm{~m} / \mathrm{s}$ which is the minimum sink rate of the aircraft. Energy harvesting flight paths will thus preferentially traverse these regions.

The wind field data extends up to $25 \mathrm{~km}$ from the surface. For practical application for soaring wind fields at altitudes of close proximity to the ground are required. Thus from the data, wind fields are calculated by interpolation at an altitude of $1000 \mathrm{~m}$ and $2000 \mathrm{~m}$ as shown in Figure 11. Contours slice of wind at two different altitudes are shown. Contours slices show the magnitude of the vertical component of the wind field. The green surface at the bottom shows the terrain of the region of Central Pennsylvania. The two translucent contour plots shows the vertical component of wind at two different altitudes.

Paths from eight starting points distributed around the domain to a goal located near $(40,45)$ are shown in Figure 12. For all paths the weight function was set to the value $\alpha_{\text {critical }}=0.96$ found using the simplified wind fields.

Figure 12 shows $A^{*}$ paths to goal from eight different starting locations to the goal for two altitudes. Both the figures show similar trends given the similar wind fields. In Figure 12(a) paths originating in the north and northwest corners merge in the middle to regions of favorable wind. Similarly two paths which originate in the south and south-east corner of the map cross a narrow region of downwards moving air and then joins before moving on to the goal.

Figure 12(b) also shows similar "corridors" in the results. Two paths which originate in the south and 


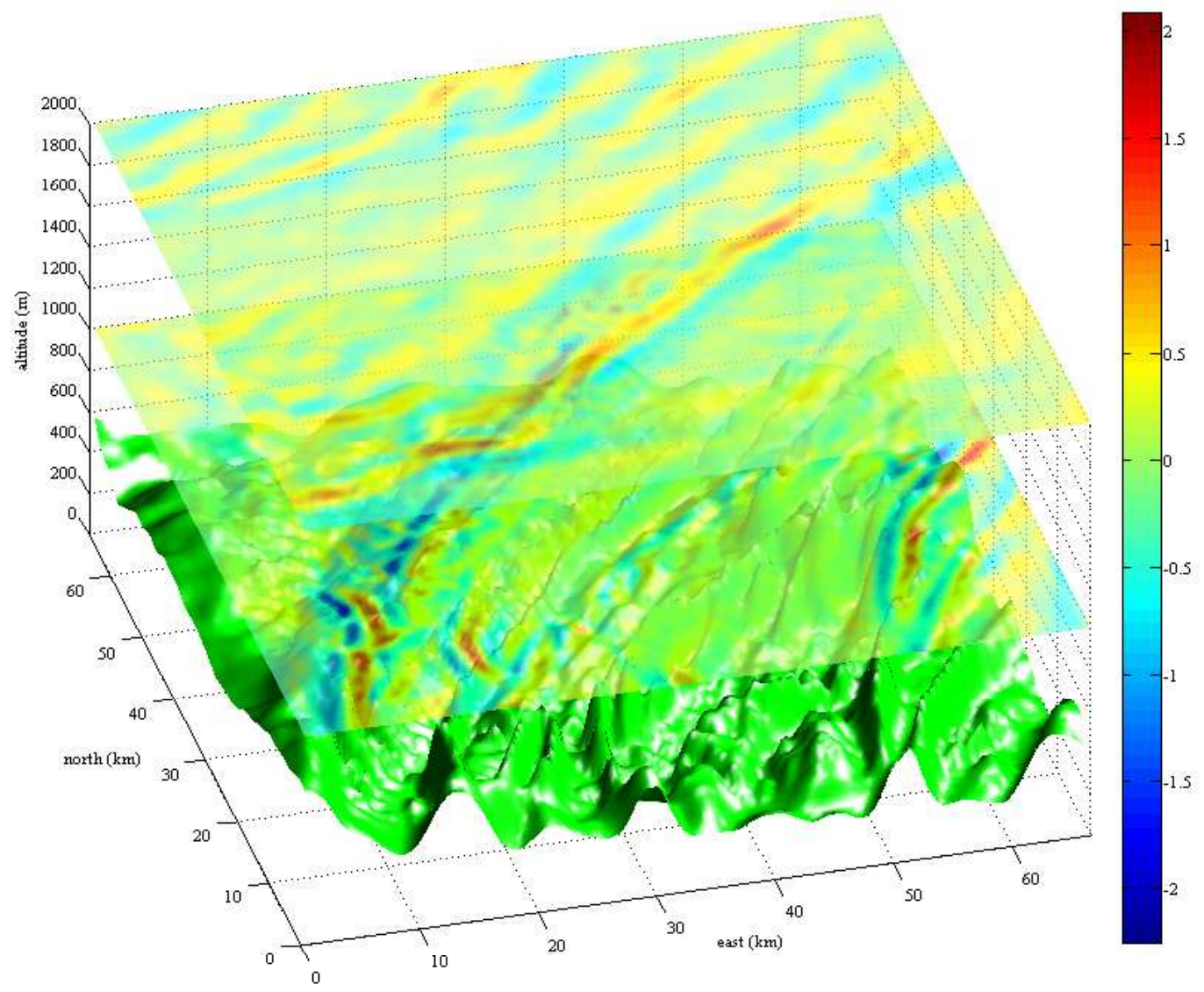

Figure 11. Contour plot of vertical component of wind field at 0700 UTC at altitude of $2 \mathrm{~km}$

south west corner merge in the region of strong upward moving air. Paths originating from the south west also merge in this path near just before the goal. Path originating from the north-west faithfully follows regions of relatively upward moving air while avoiding any downward moving air. Finally paths originating from north and northeast joins the other two just before reaching the goal. Thus routes for the given wind field data that vehicles should follow to optimize their energy requirement and time to reach the goal are identified. Qualitatively the paths follow regions of upwards moving air, but there are some cases where energetically more favorable paths would seem to exist. For example the path starting from south west corner in Figure 12(a) should have followed the more favorable wind and merged with the path originating from the bottom middle

\section{Conclusion}

This paper has presented an approach to soaring trajectory generation based on an $\mathrm{A}^{*}$ algorithm which uses a weighted sum of required energy and distance to goal as the cost function.

The speed to fly over an edge in the graph is computed by minimizing the energy expenditure for that segment, including the effects of three dimensional wind. Regeneration (conversion of potential or kinetic energy to stored electrical energy using a windmilling propeller or ram air turbine) is included, thus the net required energy for flying a path to the goal can be computed. A critical parameter is the value of weight which balances energy expenditure versus progress to goal: improper choice can result in either energetically unfavorable paths or in paths which meander without sufficient progress to goal. 


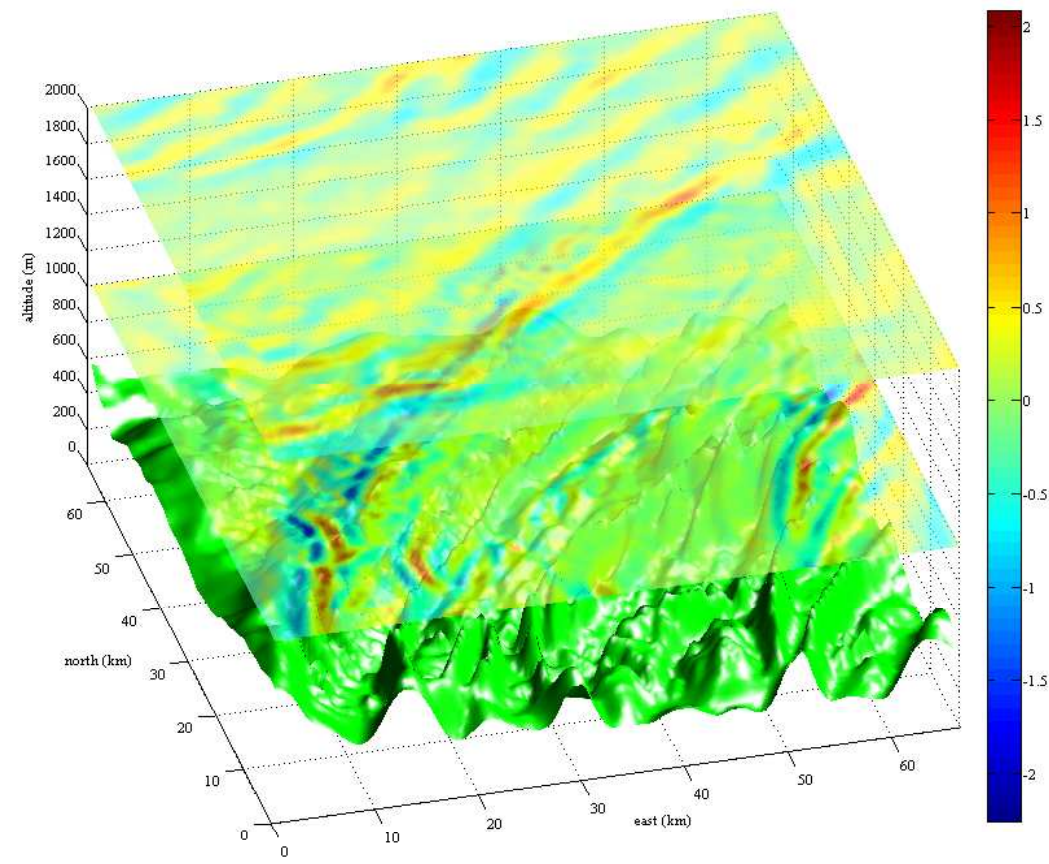

(a) A* Paths to reach the goal over wind field at 0700 UTC at altitude of $1 \mathrm{~km}$

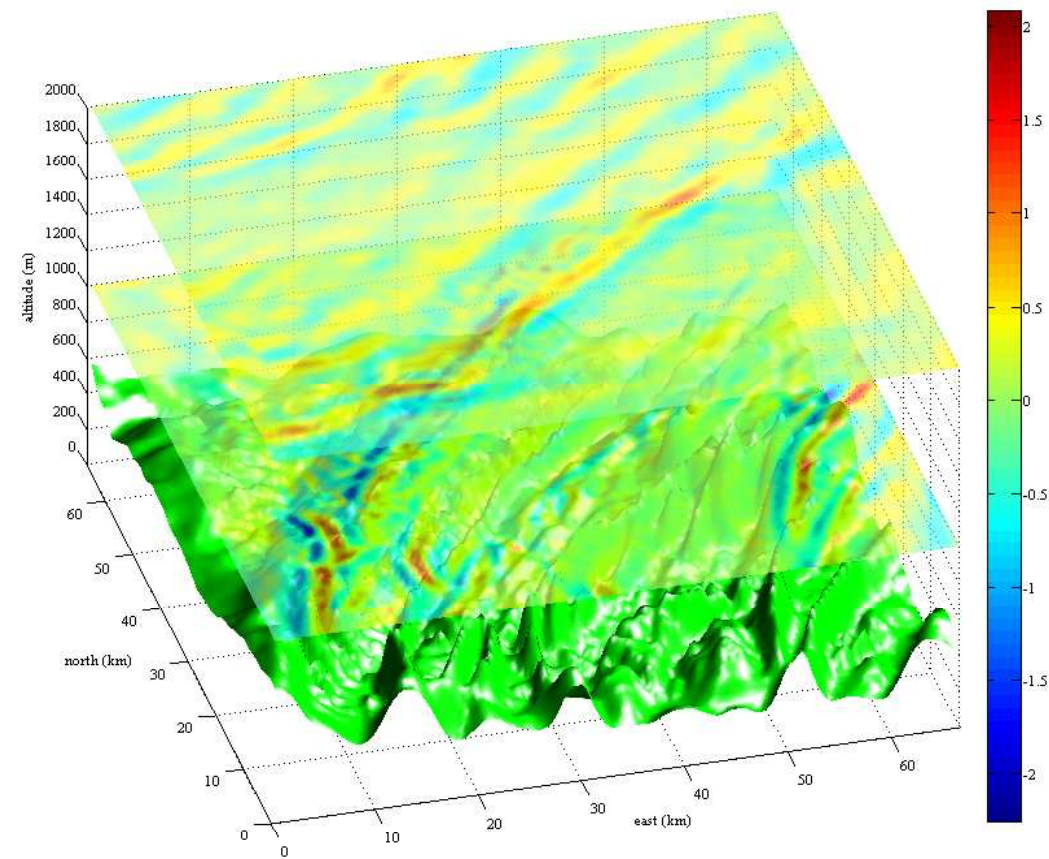

(b) $\mathrm{A}^{*}$ Paths to reach the goal over wind field at 0700 UTC at altitude of $2 \mathrm{~km}$

Figure 12. A* Paths to reach the goal over wind field at 0700 UTC at two different altitudes 
Scenarios involving simplified wind fields (a thermal-like wind field and a ridge wind field computed using potential flow) were used to find a good value of the weighting factor. For the scenarios examined here a sharp jump occurs in planned trajectories once the weight parameter reaches a critical value. After this jump flight paths follow the route of maximum energy gain without further refinement for reducing time to goal. This suggests that there is a maximum practical value of weight beyond which flight paths, while feasible, are not particularly useful.

Finally this paper applied the $\mathrm{A}^{*}$ approach to flight path planning in a realistic wind field computed using a high-fidelity forecasting tool. Given a wind filed this method enables us to find energy efficient routes for high endurance flight paths.

\section{Acknowledgments}

This research was funded by the National Science Foundation under Grant IIS-0746655. The wind field data of Section V was provided by George S. Young, Brian J. Gaudet, Nelson L. Seaman and David R. Stauffer of the Penn State University Department of Meteorology.

\section{References}

${ }^{1}$ MacCready Jr., P. B., “Optimum Airspeed Selector," Soaring, January-February 1958, pp. 10-11.

${ }^{2}$ Cochrane, J. H., "MacCready Theory with Uncertain Lift and Limited Altitude," Technical Soaring, Vol. 23, No. 3, July 1999, pp. 88-96.

${ }^{3}$ Reichmann, H., Cross-Country Soaring, Thomson Publications, Santa Monica, California, 1978.

${ }^{4}$ Arho, R., "Optimal Dolphin Soaring as a Variational Problem," OSTIV Publication XIII, Organisation Scientifique et Technique Internationale du Vol à Voile, 1974.

${ }^{5}$ Metzger, D. E. and Hedrick, J. K., "Optimal Flight Paths for Soaring Flight," Journal of Aircraft, Vol. 12, No. 11, 1975, pp. $867-871$.

${ }^{6}$ Sandauer, J., "Some Problems of the Dolphin-Mode Flight Technique," OSTIV Publication XV, Organisation Scientifique et Technique Internationale du Vol à Voile, 1978.

${ }^{7}$ de Jong, J. L., "The Convex Combination Approach: A Geometric Approach to the Optimization of Sailplane Trajectories," OSTIV Publication XVI, Organisation Scientifique et Technique Internationale du Vol à Voile, 1981, pp. 182-201.

${ }^{8}$ Pierson, B. L. and Chen, I., "Minimum Altitude Loss Soaring in a Specified Vertical Wind Distribution," NASA Conference Publication 2085, Science and Technology of Low Speed and Motorless Flight, edited by P. W. Hanson, NASA, Hampton, Virginia, March 1979, pp. 305-318.

${ }^{9}$ Sander, G. and Litt, F. X., "On Global Optimal Sailplane Flight Strategy," NASA Conference Publication 2085, Science and Technology of Low Speed and Motorless Flight, edited by P. W. Hanson, NASA, Hampton, Virginia, March 1979, pp. $355-376$.

${ }^{10}$ Allen, M. J., "Autonomous Soaring for Improved Endurance of a Small Uninhabited Air Vehicle," 43rd AIAA Aerospace Sciences Meeting and Exhibit, American Institute of Aeronautics and Astronautics, Reno, Nevada, January 2005.

${ }^{11}$ Allen, M. J. and Lin, V., "Guidance and Control of an Autonomous Soaring Vehicle with Flight Test Results," AIAA Aerospace Sciences Meeting and Exhibit, AIAA Paper 2007-867, American Institute of Aeronautics and Astronautics, Reno, Nevada, January 2007.

${ }^{12}$ Edwards, D. J., "Implementation Details and Flight Test Results of an Autonomous Soaring Controller," AIAA Guidance, Navigation and Control Conference, American Institute of Aeronautics and Astronautics, Reston, Virginia, August 2008.

${ }^{13}$ Torroella, J. C. R., Long Range Evolution-based Path Planning for UAVs through Realistic Weather Environments, Master's thesis, University of Washington, Seattle, Washington, 2004.

${ }^{14}$ Jardin, M. R. and Bryson, A. E., "Neighboring Optimal Aircraft Guidance in Winds," Journal of Guidance, Control and Dynamics, Vol. 24, No. 4, 2001, pp. 710-715.

15 "MM5 Community Model Homepage," .

${ }^{16}$ Langelaan, J. W., "Tree-Based Trajectory Planning to Exploit Atmospheric Energy," Proceedings of the American Control Conference, Seattle, Washington, June 2008.

${ }^{17}$ Langelaan, J. W., "Long Distance/Duration Trajectories for Small UAVs," AIAA Guidance, Navigation and Control Conference, American Institute of Aeronautics and Astronautics, 2007.

${ }^{18}$ Chakrabarty, A. and Langelaan, J. W., "Energy Maps for Long-Range Path Planning for Small- and Micro - UAVs," AIAA Guidance, Navigation and Control Conference, AIAA Paper 2009-6113, American Institute of Aeronautics and Astronautics, Chicago, Illinois, 2009.

${ }^{19}$ MacCready Jr., P. B., "Regenerative Battery Augmented Soaring (article)," Journal of Technical Soaring, Vol. XXIII, No. 1, January 1999. 


\section{Appendix: Vehicle Properties}

Simulation results are based on the $\mathrm{RnR}$ products $\mathrm{SB}-\mathrm{XC}$ radio control glider.

Note that a fourth order polynomial is used to relate $C_{D}$ to $C_{L}$ : this provided a better fit to the computed data over the full speed range.

Table 1. Parameters for SB-XC glider.

\begin{tabular}{ccc}
\hline \hline variable & value & description \\
\hline $\mathrm{m}$ & $10 \mathrm{~kg}$ & mass \\
$\mathrm{S}$ & $1 \mathrm{~m}^{2}$ & wing area \\
$f\left(C_{L}\right)$ & $0.1723 C_{L}^{4}-0.3161 C_{L}^{3}+0.2397 C_{L}^{2}$ & \\
& $-0.0624 C_{L}+0.0194$ & \\
$v_{a, \text { min }}$ & $12 \mathrm{~m} / \mathrm{s}$ & \\
$v_{a, \text { max }}$ & $35 \mathrm{~m} / \mathrm{s}$ & efficiency of the propeller \\
$\eta_{p}$ & 0.80 & efficiency of motor \\
$\eta_{m}$ & 0.90 & efficiency of speed controller \\
$\eta_{e s c}$ & 0.95 & \\
\hline \hline
\end{tabular}

